\title{
QUALITATIVE SUPERVISION OF NAVAL DIESEL ENGINE TURBOCHARGER SYSTEMS
}

\author{
J.M. Marchal* and E.F. Camacho** \\ *Esc. Superior de la Marina Civil, Universidad de Cadiz, Spain \\ **Escuela Superior de Ing. Industriales, Universidad de Sevilla, Spain
}

\begin{abstract}
This paper presents a qualitative model the diesel engine turbocharger system of a ship. The paper also shows how qualitative models can be use for an intelligent monitoring of the process concerned.
\end{abstract}

Keywords: Qualitative modelling, causal propagation, supervision, engine monitoring.

\section{INTRODUCTION}

One of the most useful fields of application of expert systems is intelligent monitoring of complex processes. Intelligent monitors are supposed to assist control center operators by performing, among other things, the following functions:

1. Presenting relevant information about the present state of the process to the operator.

2. Diagnosing faults, if any, that lead the process to its present state.

3. Predicting possible future states or faults if certain actions are (are not) taken.

4. Giving advice about possible actions to be taken.

All these functions need analysis and interpretation of sensor data to determine their meaning in order to explain what is (or may be) taking place in the process. It is clear that this type of interpretation must be based on a profound knowledge of the process. Two things are needed for this:

- A representation of the knowledge of the process.

- The possibility of reasoning with this knowledge.

The knowledge of a process can be represented by heuristic rules and/or by models (TravéMasuyés,1990). The interest of models for knowledge representation is their inherent possibilities for reasoning (Davis and Hamscher,1988). This must comprise not only knowledge of the separate parts, but also of how they are connected and about how they work together.

Object-oriented programming languages are good tools for representing this type of knowledge. Concepts of parts, components and their relationships are easily coded in these types of languages. On the other hand, Qualitative Simulation (Kuipers,1986) seems to be the appropiate technique to perform the causal reasoning needed in some of the functions mentioned above.

This paper presents a qualitative model of a ship diesel engine system. The paper also shows how Qualitative Modelling can be used for an intelligent monitoring of the process concerned. Artificial Intelligence Techniques have been applied to ship engine monitoring in the past (Katsoulakos et al., 1989), although the approach used was rule based and did not consider qualitative behaviour. The main objective of this work is to develop qualitative behaviour models of the main engine a ship that could be used for monitoring, failure detection, diagnosis, prediction and instruction.

The system developed has been implemented in SMALLTALK (Goldberg and Robson,1984), which is a general purpose object-oriented language and thus allows Qualitative Simulation and time causal reasoning to be integrated into a more general reasoning system.

The paper is organized as follows. Section 2 describes the process concerned and the model developed. Section 3 is dedicated to the qualitative propagation algorithm while section 4 explains how the object - oriented approach has been used. Some simulation runs showing malfunction detection are presented in section 5. Section 6 is dedicated to presenting some concluding remarks.

\section{MODEL DESCRIPTION}

The engine room of a ship considered is composed of the following subsystems: The main engine, the main engine turbocharger, the auxiliary engine, the lubricating subsystem, the salt water system, the combustion feeding system, the compressed air system etc. The engine room of a ship is a fundamental part of the same and has, therefore, to be continuously supervised.

The process considered, see figure 1 , corresponds to 


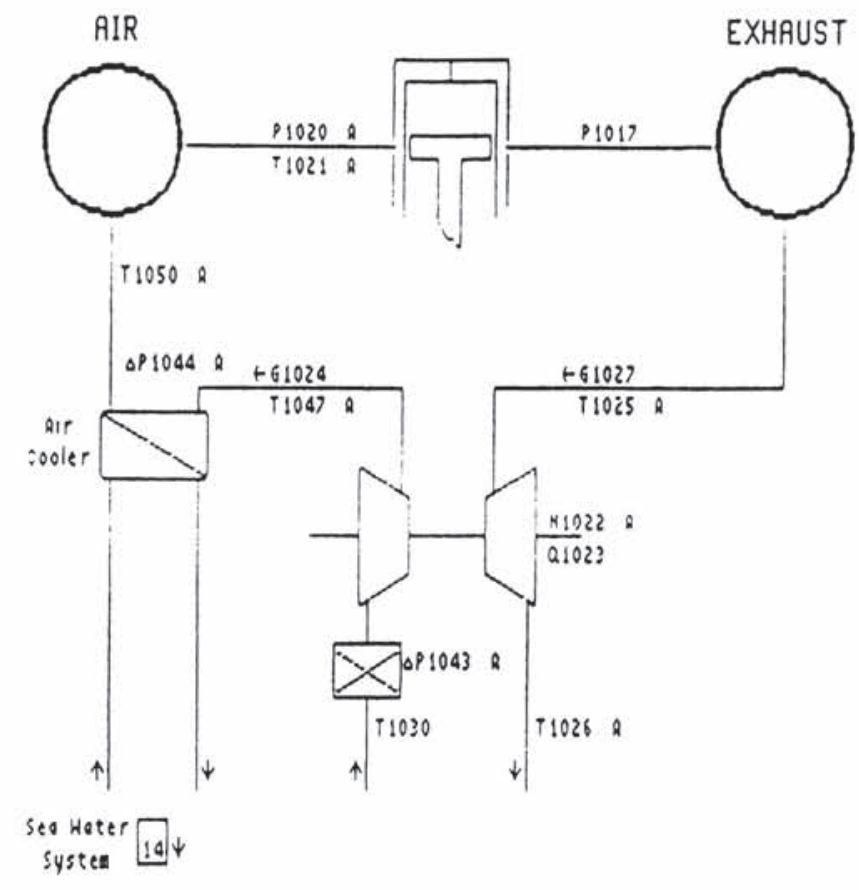

Figure 1: Ship main engine turbocharger system

a ship main engine turbocharger system. Naturally aspirated engines draw air of the same density as the ambient atmosphere and this density determines the maximum weight of fuel that can be burned in the cyclinders and therefore the maximum power obtained. If the air density is increased, by a compressor, the amount of air is increased and more weight of fuel can be effectively burned and the power developed also increases. This procedure is implemented in most modern diesel engines by using exhaust gas turbocharging where exhaust gases are used to power the compressor. A substancial amount of the total heat energy is wasted to the exhaust gases, and although it is relatively inexpensive to drive the compressor directly from the engine by gear, an increase in power is obtained by using the exhaust gases to drive the compressor.

The inlet air is filtered and goes through the compressor. As the temperature of the inlet air after being compressed is too high to go into the cylinders, it has to be cooled down. This is accomplished by an air cooler using sea water as a coolant. Some of the surplus energy of the exhaust gases is used to power the turbine coupled with the compressor as indicated before.

Temperatures of gases in ship diesel engines are very valuable sources information for monitoring their conditions. A model of the behaviour of the gases was considered to be a good tool for the supervision of ship engine rooms. In this sense, a qualitative model of the turbocharger subsystem was developed. A modular and hierarchical decomposition of the system was established. This way of representing the system adapts to physical reality, topology, the operator's mental models and allows for easy generalization when representing the global complexity of a ship engine room.

The main parts of the model presented are:
- Filters

- Air Cooler

- Turbine-compressor

- Cylinders

- Receiver

The main variables taken into account by the model are: cylinder inlet air pressure and temperature, aircooler inlet air temperature and pressure drop, seawater inlet temperature, turbine and compressor temperatures and exhaust pressure and temperatures.

All these components have been modelled according to Kuipers (1984) although the idea used for their aggregation in order to form the the system is nearer to the component ontology used by De Kleer (1984). The same applies to the concepts of connections, causality and heuristics used.

The models have been obtained using physical laws and heuristic rules, given by the experts. The heuristic rules are used to resolve the ambiguities originated in the qualitative simulation. Notice that the models given corresponds to quasi stationary conditions.

Fig 2 shows the model of the air cooler. The difference (DtTaw) of inlet air temperature $(T i E)$ and sea water temperature ( $T s w i)$ multiplied by the heat transfer coefficient determines the heat flow $(Q E)$. The heat flow is also related to the air flow (GairE) and the difference $(D t T E)$ between inlet air temperature $(T i E)$ and outlet air temperature $(T o E)$. The air flow is also related to the difference (DTPE) between the inlet air pressure $(P i E)$ and outlet air pressure $(P o E)$ as indicated.

The qualitative model of the cylinders can be seen in Fig. 3 The air flow (Gair) multiplied by the fuel to air ratio $(R m)$ will determine the oil consumption $(G c b)$, 


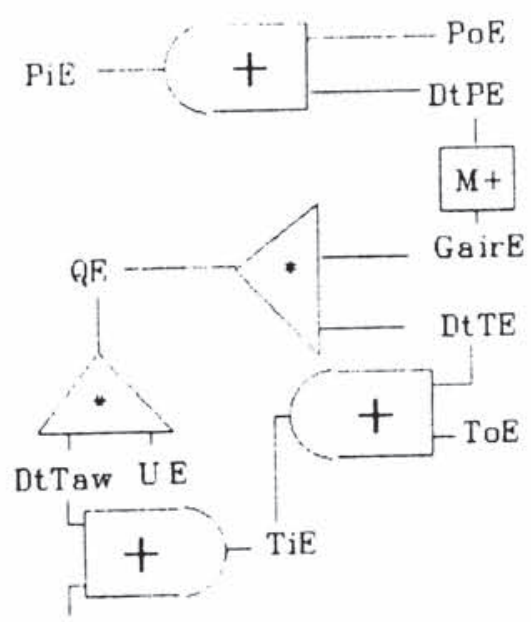

Tswi

Figure 2: Cooler Model

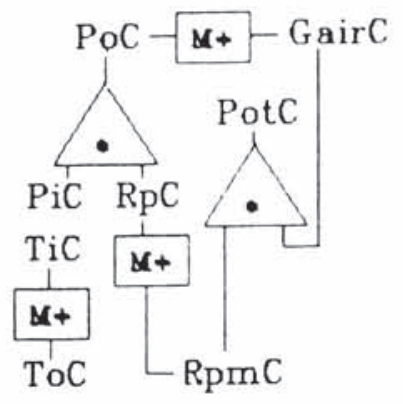

Figure 4: Compressor Model

which in turn if multiplied by the cylinder efficiency $(E f)$ will generate the output power $(P o t)$. The exhaust gas flow $(G g)$ is related to the air flow and the difference $(D t T c b)$ of the gases $(T g C y l)$ and air inlet temperature $(\mathrm{TiCyl})$ as shown.

Figure 4 shows the qualitative model of the compressor. The power $(P o t C)$ depends of the product of the compressor speed $(R p m C)$ and the air flow ( $\operatorname{Gair} C$ ) through the compressor, which in turn is related monotonically to the compression ratio $(R p C)$. Inlet $(P i C)$ and outlet $(P o C)$ air pressures are related by the compression ratio. Inlet $(\mathrm{TiC})$ and outlet $(\mathrm{ToC})$ air temperatures are also related monotonically.

\section{QUALITATIVE PROPAGATION BETWEEN COMPONENTS.}

We consider a system to be a collection of interconnected components and the interaction between them to be a form of directional causality. Thus the behaviour of the system is obtained from the behaviour of each component and the connections between them (Williams,1990)

The qualitative simulation (Kuipers 1984; Kuipers 1986) begins with the propagation of the known information, or known disturbances, to the system through the constraints, in order to complete the description of the direction of change for each variable, at a given time-point.

The algorithm for the propagation of a disturbance in a component to the whole system is composed of the followings steps:

1 Set initial conditions.

2 Form a queue of components to be propagated (QCP) with the component which caused disturbances.

3 While queue QCP is not empty repeat:

3.1 Get the first component of the queue QCP. Name this the active component. Remove it from queue QCP.

3.2 For the active component propagate through the constraints of its qualitative models the information known about it.

3.3 If there is any variable with ambiguity at the end of the propagation process use 
heuristic rules to solve it and propagate the results.

3.4 Get the possible states for active component and filter them by testing the consistency with the neighboring components in the system, using topology.

3.5 Using the connections apply causality to propagate the changes in the shared variables with other components and add these components to the QCP queue.

\section{OBJECT-ORIENTED APPROACH.}

An object-oriented approach has been used to implement the model, to propagate disturbances and for qualitative simulation. This approach has the following advantages:

- modularity, with its possibility of description of complex systems.

- hierarchy of models and levels of description.

- good framework for the natural description of components.

- easy developement of prototypes.

A version of the QSIM algorithm for qualitative simulation, proposed by Kuipers (Kuipers 1986), has been implemented in SMALLTALK-V2 language.

$\mathrm{T}$ developed are the following:

A brief description of the more important classes developed is given in the following:

System Class. This establishes the set of component models and connections which define the system. It expresses the topology of the system, the aggregation of the components and the hierarchical organisation between them.

Model Class. This contains the set of variables and constraints which caracterize the structure of the model of a component.

Conection Class. This class expresses the causality and the link between components. It supports the continuity and compatibility conditions (conservation of matter for fluid systems, conservation of energy for thermal system,...) (De Kleer and Brown, 1984).

Variable Class. The objects of this class are variables, continuous real-valued functions of time, with a qualitative value and tendency, an ordered value space and a set of possible transitions from their current qualitative state.

Constraint Class. This contains a hierarchy of the different kind of constraints between variables.

Transition Class. This class describes the two types of qualitative state transitions for a variable:
P-transitions, moving from a point to an interval, and I-transitions, moving from an interval to a point.

Tuple Class. Each object of this class contains the set of pairs and triples generated for each constraint with the transitions of its arguments.

State Class. Each object of this class is the qualitative state of a model and its qualitative time description ( interval or time-point).

Interpretation Class. The object of this class are the possible new qualitative states resulting from applying the QSIM algoritm.

Prediction Class. This class contains the objects resulting from qualitative simulation. They are composed of an initial state and the set of states the model can reach until a distinguished time-point.

\section{APPLICATION EXAMPLE}

As an application example the effect of a cooler soiling process on the diesel engine is analyzed. The engine speed is considered to be constant and the air filter is not taken into account for the sake of clarity.

The initial value of each variable is set by a landmark (written between brackets), which defines a qualitative value for the time points or by a pair of landmarks for the time intervals. Thus, the air flow in the cylinders GairCyl get a landmark value (Gairt) corresponding to to the full ahead engine regime. This landmark is placed in the following ordered space:

$$
\text { [ zero....Gairst.....Gairt..... inf ] }
$$

where: Gairst is the minimal air flow to ensure a complete combustion. So if the air flow is less than Gairst the perfomance of the engine decreases and a different qualitative region is reached.

We consider a full ahead quasi-static engine regime. The engine speed, the efficiency of the combustion process, the efficiency of the turbine, the input sea water temperature and the input compressor temperature are supposed to be constant for this qualitative state. This is a common situation. The air cooler soiling process is represented by assigning a decreasing tendency to the coefficient of heat transfer.

The initial situation of the air cooler is given by:

\begin{tabular}{|l|c|c|c|} 
Variable & Name & Value & Tend. \\
\hline \hline Inlet air temp. & TiE & $($ ToCt $)$ & std \\
\hline Inlet sea water temp. & Tswi & $(T s w i O)$ & std \\
\hline Heat transfer coeff. & $U E$ & $(U E O)$ & dec \\
\hline Inlet air pressure & $P i E$ & $(P o C t)$ & std \\
\hline
\end{tabular}

From this situation it can be inferred by propagation that the heat flow in the cooler decreases. By applying the heuristic :

if the heat flow $Q E$ changes, then the temperature difference DtTE in the cooler will change in the same direction

Thus, we obtain: 


\begin{tabular}{|l|l|l|l|}
\hline air temp. diff. & $D t T E$ & (DtTEO) & dec \\
\hline
\end{tabular}

that being propagated implies:

\begin{tabular}{|l|l|l|l|}
\hline cooler outlet air temp. & ToE & $(T o E t)$ & inc \\
\hline
\end{tabular}

Within this situation there are three possible states for the cooler that correspond to the ambiguity in air flow GairE.

a)

\begin{tabular}{|l|l|l|}
\hline Gair $E$ & (Gairt) & inc \\
\hline PoE & (PoEt) & dec \\
\hline ToE & (ToEt) & inc \\
\hline
\end{tabular}

b)

\begin{tabular}{|l|l|l|}
\hline GairE & (Gairt) & std \\
\hline PoE & (PoEt) & std \\
\hline ToE & (ToEt) & inc \\
\hline
\end{tabular}

c)

\begin{tabular}{|l|l|l|}
\hline GairE & (Gairt) & dec \\
\hline PoE & (PoEt) & inc \\
\hline ToE & (ToEt) & inc \\
\hline
\end{tabular}

After checking the consistency of each possibility in the receiver model it is noted that c) is the only one that is consistent with it.

The output variables of the cooler (pressure $P o E$, temperature $T o E$ and air flow Gair $E$ ) are inputs for the receiver model, and this implies:

\begin{tabular}{|l|l|l|l|}
\hline air density & Dair & (Dairt) & dec \\
\hline
\end{tabular}

The cylinder variables already defined, for being shared with the receiver are:

\begin{tabular}{|l|c|c|c|}
\hline cylinders inlet air temp. & TiCyl & (ToEt) & inc \\
\hline air flow & GairCyl & (Gairt) & dec \\
\hline
\end{tabular}

Taking into account that for this qualitative region the efficiency of the cylinder is kept constant as well as the power PotM, the cylinder model implies:

\begin{tabular}{|l|l|l|l|}
\hline exhaust gas flow & $G g$ & $(G g t)$ & dec \\
\hline exhaust gas temp. & $T g$ & $(T g t)$ & inc \\
\hline
\end{tabular}

In the turbine we can deduce that the power Pot T decreases by applying, apart from the qualitative model, the heuristics: a) if the exhaust gas flow changes, then the power will change in the same direction.

b) if the exhaust gas temperature changes, then the temperature difference between input and output in the turbine will change in the same direction.

The latter is of the KVL type of heuristic proposed by De Kleer (De Kleer 1984)

In the compressor the variables which have already been stated by their connections with the cooler and the turbine are:

\begin{tabular}{|l|l|l|l|}
\hline air flow & GairC & (Gairt) & dec \\
\hline power & PotC & (Pot Tt) & dec \\
\hline
\end{tabular}

The input temperature is considered constant and, for the supposed regime, the influence of a change in the compression ratio $R p C$ upon the output temperature To $C$ has been excluded. In the compressor we have applied the heuristic:

if the power PotC changes, then the air flow and the speed $R p m C$ will change in the same direction.

The compressor model implies:

\begin{tabular}{|l|l|l|l|}
\hline comp. outlet air pressure & $P o C$ & $(P o C t)$ & $d e c$ \\
\hline
\end{tabular}

The compressor-cooler connection implies that the input pressure of the latter $P i E$ changes its initial tendency of (std) to dec, so that it is necessary to propagate with the new tendency, and apply a new heuristic:

if the input cooler pressure PiE changes, then the output cooler pressure will change in the same direction.

With this heuristic a new tendency $(d e c)$ is obtained for the pressure in the receiver (it was inc before) that is consistent with its qualitative model and that does not produce new changes in the rest of the components. To summarize, the new situation of the main engine turbo-charger system can be characterized by the following variables:

\begin{tabular}{|l|c|c|c|}
\hline air receiver pressure & PCol & $(P o E t)$ & dec \\
\hline turbine speed & $R p m T$ & $(R p m T t)$ & dec \\
\hline $\begin{array}{l}\text { exhaust gas temp. } \\
\text { inlet turbine }\end{array}$ & TiT & $(T g t)$ & inc \\
\hline $\begin{array}{l}\text { exhaust gas temp. } \\
\text { outlet turbine }\end{array}$ & $T o T$ & $(T o T t)$ & inc \\
\hline comp. inlet air temp. & TiC & $(T s m)$ & std \\
\hline cooler inlet air temp. & TiE & $(T o C t)$ & std \\
\hline cooler outlet air temp. & ToE & $(T o E t)$ & inc \\
\hline air receiver temp. & TCol & $(T o E t)$ & inc \\
\hline air flow & Gair & (Gairt) & dec \\
\hline cooler diff. pressure & $D t P E$ & $(D t P E t)$ & dec \\
\hline
\end{tabular}

Thus, we have an initial state for the qualitative simulation of all the components. As we are at a time-point the next qualitative state will be a time-interval, where the variables will be reach their next landmarks (for example Gairst for the air flow Gair). The new qualitative states will appear and we must change some qualitative models (for example the cylinder model if 
Table 1: Numerical Results Obtained with the Simulator

\begin{tabular}{|l|c|c|c|}
\hline & & $\begin{array}{c}\text { clean } \\
\text { cooler }\end{array}$ & $\begin{array}{c}\text { dirty } \\
\text { cooler }\end{array}$ \\
\hline air receiver pressure & PCol & $1.603 \mathrm{bar}$ & $1.410 \mathrm{bar}$ \\
\hline turbine speed & $R p m T$ & $6535 \mathrm{rpm}$ & $6039 \mathrm{rpm}$ \\
\hline exhaust gas temp. inlet turbine & $\mathrm{TiT}$ & $360.8^{\circ} \mathrm{C}$ & $420.8^{\circ} \mathrm{C}$ \\
\hline exhaust gas temp. outlet turbine & $\mathrm{ToT}$ & $269.8^{\circ} \mathrm{C}$ & $329.8^{\circ} \mathrm{C}$ \\
\hline compressor inlet air temp. & $\mathrm{TiC}$ & $36.7^{\circ} \mathrm{C}$ & $36.9^{\circ} \mathrm{C}$ \\
\hline cooler inlet air temp. & $\mathrm{TiE}$ & $129.7^{\circ} \mathrm{C}$ & $130.8^{\circ} \mathrm{C}$ \\
\hline cooler outlet air temp. & $\mathrm{ToE}$ & $35.9^{\circ} \mathrm{C}$ & $62.9^{\circ} \mathrm{C}$ \\
\hline air receiver temp. & $T C o l$ & $43.7^{\circ} \mathrm{C}$ & $71.2{ }^{\circ} \mathrm{C}$ \\
\hline air flow & $\mathrm{Gair}$ & $14.02 \mathrm{ton} / \mathrm{h}$ & $11.81 \mathrm{ton} / \mathrm{h}$ \\
\hline cooler diff. pressure & $\mathrm{DtPE}$ & $128 \mathrm{mmW}$ & $91 \mathrm{mmW}$ \\
\hline
\end{tabular}

the air flow Gair reaches the Gairst landmark). However, in monitoring tasks on ships these qualitative changes are not usually allowed to happen, and it is more important to identify the previous disturbance process.

Some numerical simulation tests were carried out in the DPS 100 of NORTHCONTROL trainning simulator in order to in order to verify the results obtained by the qualitative simulation. The results of the numerical simulation are indicated in table I. The information given in the table corresponds to the numerical values of the main variables, in a quasi stationary regime, when working with a clean and a dirty cooler. As can be seen, the numerical results agree with the qualitative results given before.

\section{CONCLUSIONS}

Qualitative modelling is a good alternative for tasks as supervision, diagnosis, instruction, and, in general those that can be aided by techniques based upon knowledge.

The application of heuristic rules would has shown to be very useful in reducing the ambiguities of the qualitative models. Several examples have been presented. In the same way the relationships between compatibility and consistency, that can be established among the components, through the connections, reduces these ambiguities.

The object oriented approach has shown to be quite adequate to represent complex systems, and a structure of classes for qualitative simulation has been proposed.

\section{ACKNOWLEDGEMENT}

The authors would like to acknowledge CICYT for funding this work.

\section{REFERENCES}

Davis R. and W. Hamscher (1988) Model-based reasoning: troubleshooting, in H.E. Shrobe (Ed.). Exploring Artificial Intelligence, Chap.8.Morgan Kaufmann, San Mateo, California, pp 297-347.

De Kleer J.(1984). How circuit work, in D.G. Bobrow (Ed.) Qualitative Reasoning About Physical Systems. North-Holland, Amsterdam, pp 205-280.

De Kleer J. and J.S Brown (1984). A qualitative physics based on confluences, in D.G. Bobrow (Ed.) Qualitative Reasoning about Physical Systems. North-Holland, Amsterdam, pp 7-84.

Goldberg A.] and D. Robson (1984) Smalltalk-80: The language and its Implementation. Reading, MA. Addison-Wesley.

Katsoulakos P.S., J.Newland, J.T. Stansfield and T. Ruxton (1989) Monitoraggio, Raccolta Dati e Sistemi Esperti per la Diagnostica delle Avarie di Machina, Tecnologie per il Mare, L'Automazione Navale, pp 38-42, march.

Kuipers B. (1984) Commonsense reasoning about causality: deriving behaviour from structure, in D.G. Bobrow (Ed.) Qualitative Reasoning About Physical Systems. North-Holland, Amsterdam. pp 169-204.

Kuipers B. (1986) Qualitative simulation, Artificial Intelligence $29,289-338$.

Travé-M asuyés L.,A. Missier and N.Piera (1990) Qualitative models for automatic control process supervision, Proc. of the $11^{\text {th }}$ IFAC World Congress, Vol. 7, pp 198-203, Tallin, Estonia.

Williams B.C. (1990) Temporal qualitative analysis: explaining how physical systems work, in D.S. Weld and J. de Kleer (Ed.). Readings in Qualitative Reasoning About Physical Systems, Chap.2. Morgan Kaufmann, San Mateo. California. pp 133-177. 